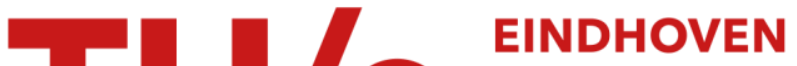 UNIVERSITY OF TECHNOLOGY
}

\section{Simulation of electrical conductivity in a pi-conjugated polymeric conductor with infrared light}

\section{Citation for published version (APA):}

Meskers, S. C. J., Duren, van, J. K. J., \& Janssen, R. A. J. (2002). Simulation of electrical conductivity in a piconjugated polymeric conductor with infrared light. Journal of Applied Physics, 92(12), 7041-7050.

https://doi.org/10.1063/1.1519948

DOI:

10.1063/1.1519948

Document status and date:

Published: 01/01/2002

\section{Document Version:}

Publisher's PDF, also known as Version of Record (includes final page, issue and volume numbers)

\section{Please check the document version of this publication:}

- A submitted manuscript is the version of the article upon submission and before peer-review. There can be important differences between the submitted version and the official published version of record. People interested in the research are advised to contact the author for the final version of the publication, or visit the $\mathrm{DOI}$ to the publisher's website.

- The final author version and the galley proof are versions of the publication after peer review.

- The final published version features the final layout of the paper including the volume, issue and page numbers.

Link to publication

\section{General rights}

Copyright and moral rights for the publications made accessible in the public portal are retained by the authors and/or other copyright owners and it is a condition of accessing publications that users recognise and abide by the legal requirements associated with these rights.

- Users may download and print one copy of any publication from the public portal for the purpose of private study or research.

- You may not further distribute the material or use it for any profit-making activity or commercial gain

- You may freely distribute the URL identifying the publication in the public portal.

If the publication is distributed under the terms of Article $25 \mathrm{fa}$ of the Dutch Copyright Act, indicated by the "Taverne" license above, please follow below link for the End User Agreement:

www.tue.nl/taverne

Take down policy

If you believe that this document breaches copyright please contact us at:

openaccess@tue.nl

providing details and we will investigate your claim. 


\title{
Stimulation of electrical conductivity in a $\pi$-conjugated polymeric conductor with infrared light
}

\author{
S. C. J. Meskers, ${ }^{\text {a) }}$ J. K. J. van Duren, and R. A. J. Janssen \\ Laboratory for Macromolecular and Organic Chemistry, Eindhoven University of Technology, P.O. Box 513, \\ NL-5600MB Eindhoven, The Netherlands
}

(Received 17 June 2002; accepted 16 September 2002)

\begin{abstract}
Irradiation with infrared light is found to stimulate the electrical conductivity of a film of an organic polymeric conductor [poly(3,4-ethylenedioxythiopene) with polystyrene sulfonate]. The change in conductivity is found to be linear in the intensity of the irradiation $\left(4-400 \mathrm{~mW} / \mathrm{cm}^{2}\right)$. Both frequency and time domain measurements reveal that the change in resistance induced by irradiation, relaxes according to $\Delta R(t) \propto(1 / t)^{0.6}$, with $t$ as the time after excitation. As a possible mechanism for this relaxation, we model the diffusion of heat from the polymer film to the supporting glass substrate. Assuming that the change in resistance is linear with the raise in temperature caused by the infrared irradiation, one predicts a $\Delta R(t) \propto(1 / t)^{0.5}$ dependence. The similarity between the model and experimental behavior is taken as an indication that the relaxation is limited by heat transport from the polymer film and that the thermalization of the charge carriers occurs on a shorter time scale. Electrical characterization is complemented with optical measurements. These show infrared-induced transient absorption of the polymer film with practically the same relaxation behavior as the change in resistance. This suggests that the optical transients are also due to thermal excitations. In the sub-ps time domain, measurements of the change in optical transmission $(\Delta T / T)$ induced by the infrared pulse show a very short-lived component with a lifetime close to the instrumental resolution $(\sim 500 \mathrm{fs})$. The rapid response is followed by a slow component that decays according to $(\Delta T / T)(t) \propto(1 / t)^{0.65}$. This is interpreted in terms of cooling of the excited charge carriers limited by heat transport, indicating that the thermalization of the carriers occurs on the sub-ps time scale. (c) 2002 American Institute of Physics. [DOI: 10.1063/1.1519948]
\end{abstract}

\section{INTRODUCTION}

$\pi$-conjugated polymers are a relatively new class of materials that have found applications in various optoelectronic devices. Films of these materials can be rendered conductive upon (oxidative) doping. ${ }^{1}$ Doping with oxidizing agents generally does not produce conductive materials that are environmentally stable. Dedoping followed by a loss of conductivity usually occurs quite rapidly. However, a few dopantpolymer combinations are known that are extremely stable also under ambient conditions. Well known is, for instance, polyaniline doped with camphorsulfonic acid. ${ }^{2}$ In this article, we focus on another polymer-dopant combination: poly $(3,4-$ ethylenedioxythiophene ) (PEDOT) with polystyrene sulfonate (PSS). The PEDOT polymer combines a low oxidation potential with environmental stability in the oxidized state. ${ }^{3}$ In the oxidized form, positive charges on the PEDOT are balanced by negative charges on the PSS, which acts as polymeric counterion. PEDOT:PSS has found many industrial applications. ${ }^{4}$ An aqueous dispersion of these two polymers is easily processable and solid films prepared from this mixture show high conductivity (up to $600 \mathrm{~S} / \mathrm{cm}$ ). ${ }^{5}$

Photoconductivity, i.e., the change in electrical conductivity of a solid due to irradiation, can arise not only as the result of a change in carrier density but also as a result of a

\footnotetext{
a)Electronic mail s.c.j.meskers@tue.nl
}

change in carrier mobility due to absorption of radiation. ${ }^{6}$ If radiation incident on a semiconducting material is absorbed by free carriers, their energy will be increased above the value corresponding to thermal equilibrium with the lattice. In principle, this change in energy can be observed by the change in mobility and, hence, in conductivity. This type of photoconductivity can be utilized to produce sensitive receivers of electromagnetic radiation in the millimeter and infrared regions as shown by Kinch and Rollins. ${ }^{7}$ They demonstrated that $n$-doped indium antimonide at liquid-helium temperatures can be used as active material in an infrared detector with a response time of $0.1 \mu \mathrm{s}$. Another example of this type of photoconductivity was given by Rohrbacher and Karl. ${ }^{8}$ These authors studied conduction of anthracene doped with tetracene. The latter molecule acts as a hole trap. By irradiating at the infrared wavelengths corresponding to the absorption of the loaded tetracene trap (i.e., the tetracene cation) they were able to detrap the charges and collect them with help of electrodes. Later, it was shown that besides this indirect process involving an electronic excited state of the trap also direct trap-to-band transitions can be induced with infrared light. ${ }^{9}$

For PEDOT:PSS, there are infrared absorption bands associated with the charge carriers and, therefore, it is possible to excite charge carriers specifically. This may then lead to a change in conductivity similar to the cases just cited. If, however, the thermalization of the carriers is very rapid, the 
energy supplied by the photons will be dissipated very quickly and a change of conductivity due to a change in temperature will be observed. So, depending on the thermalization time of the carriers, a device consisting of a PEDOT:PSS layer with suitable electrodes may act either as a photoconductive or thermal detector for infrared light.

The main objective of this work is to study photoinduced changes in the resistivity of PEDOT:PSS and to analyze the relaxation mechanism for the excitations. Additionally, we will evaluate the feasibility of a polymeric infrared detector. A number of polymeric infrared detectors has been described in literature. Many of these involve the pyroelectric effect. ${ }^{10}$ Kaufman et al. ${ }^{11}$ have patented a polymer infrared bolometer but their work involves mainly ion implanted polymers and no description of $\pi$-conjugated polymers is given. Thus, to our knowledge, the effect on the electrical properties of excitation with infrared light involving the electronic transitions associated with the charge carriers has not been investigated for doped $\pi$-conjugated polymers.

The electrical resistivity of PEDOT doped with $\mathrm{PF}_{6}$ has been studied in detail as a function of temperature. ${ }^{12}$ Near room temperature, the resistivity shows a negative temperature coefficient $(\partial \rho / \partial T<0)$ typical for disordered conductors. The temperature dependence of the resistivity in the interval $30<T<300 \mathrm{~K}$ can be described by $\rho(T)$ $=\rho_{0} \exp \left(T_{0} / T\right)^{1 / n}$ with $n$ ranging from 3 to 4 for different films. This behavior is indicative of variable range hopping conduction. ${ }^{13}$ At low temperature $(T<10 \mathrm{~K})$, the dependence of the resistivity on temperature changes. The conductivity $\sigma$ $(=1 / \rho)$ was found to obey $\sigma(T)=\sigma_{0}+m T^{1 / 2}$, where m can have either positive or negative sign depending on the film preparation. The difference in the high- to low-temperature behavior is attributed to the increased importance of electron-electron interactions on the dynamics of the charge carriers at low temperatures. ${ }^{12}$

As a peculiar characteristic of $\pi$-conjugated polymers, the doped polymers show high cross sections for photon absorption in the midinfrared and infrared regions of the spectrum. ${ }^{1}$ The neutral form of the polymers usually does not absorb below $1.5 \mathrm{eV}$. In particular, thin films of PEDOT:PSS are almost transparent in the visible range of the spectrum and show a broad absorption band that starts at about $1.5 \mathrm{eV}$ and extends into the infrared. ${ }^{14}$ This band has been ascribed to the presence of charge carriers and has been interpreted in terms of a localization modified Drude model. ${ }^{14}$ In this model, the charge carriers in the material are regarded as a free electron gas with the distinction that the mean-free path is limited by defects related to the disorder of the polymer chains. Analysis of reflection data using this model yields the plasma frequency $\left(\omega_{\mathrm{pl}}\right)$ and a scattering time $(\tau) . \omega_{\mathrm{pl}}$ is estimated at $1.2 \mathrm{eV}$ and this energy marks the upper limit for the absorption due to intraband transitions of the free carriers associated with the translational motion of these species. The mean scattering time $\tau$, estimated at $1-10 \mathrm{fs}$, gives the average time between two consecutive collisions of a charge carrier with defects, phonons, or other carriers. This parameter can also be taken as an estimate for the lifetime of the excited state associated with the photon absorption. Apart from intraband transitions, interband and intrachain transitions may also contribute to the infrared absorption but their contribution is as yet not quantified. Studies of the polymer films at low degrees of doping ${ }^{15}$ have shown the presence of two absorption bands, one at $\sim 1.5 \mathrm{eV}$ and one at $\sim 0.5 \mathrm{eV}$. This absorption spectrum is remarkably different from that at high doping levels. For low doping levels, the plasma frequency $\left(\omega_{\mathrm{pl}}\right)$ shifts towards lower values ${ }^{16}$ so that absorption due to free carrier motion no longer contributes to the absorption in the midinfrared. At low degrees of doping, the absorption bands of the charge carriers can most likely be associated with an intrachain excitation of a carrier. This view is corroborated by recent studies on small oligomers of 3,4ethylenedioxythiophene (EDOT) in a solution where similar absorption bands due to intramolecular transitions of the radical cations have been observed. ${ }^{17}$ About the lifetime of these intrachain and/or interband excitations, almost nothing is known. If this lifetime is sufficiently long, the population of these excited states may add an additional slow component to the carrier thermalization process.

Structural studies of doped PEDOT films ${ }^{18}$ have shown that the material is largely amorphous and can be regarded as a disordered solid. In disordered materials, relaxation and thermalization of charge carriers are often slow. ${ }^{19}$ As an example, the rate of thermalization may be limited by the slow transport of carriers towards traps. In this respect, disordered conductors with a relatively high density of carriers show interesting behavior. It has been argued that due to the electrostatic interactions between the charge carriers, a Coulomb gap exists which separates the filled from the empty states available to the carriers. ${ }^{20}$ At low temperatures, excitations can have a multiparticle character and their decay can be very slow. To describe possible nonergodic behavior in the system of interacting carriers, the term "electron glass" has been coined. ${ }^{21}$

In summary, the relaxation and thermalization of photoexcited charge carriers in doped $\pi$-conjugated polymers is largely unstudied. From reflection measurements, very rapid relaxation would be expected. However, various mechanisms leading to slow relaxation rates seem feasible in disordered materials.

The organization of this article is as follows. After briefly describing the experimental details, experimental results on the influence of infrared radiation on the resistance of PEDOT:PSS will be described. We find that the changes in resistivity are accompanied by optical transients which we characterize using photoinduced absorption spectroscopy. The optical transients allow us to study dynamical processes on very short time scales. We find that, after pulsed excitation, optical and electrical transients decay according to a power law. In the discussion section, we analyze heat transport from the polymer film to the glass substrate. The decay of the temperature rise in the polymer film upon pulsed irradiation is expected to follow a power law. The predicted value for the exponent of the power law is very similar to the value observed experimentally. This strongly suggests that both the electrical and optical transients can be understood in terms of a thermal effect. Finally, we briefly evaluate the usefulness of the observed effects for detection of infrared radiation. 


\section{EXPERIMENT}

\section{A. Device preparation}

Electrode structures were deposited onto glass substrates by thermal evaporation. To improve the adhesion of the gold on the glass a thin layer of $\mathrm{Cr}$ (thickness $\sim 5 \mathrm{~nm}$ ) was first deposited followed by a thicker Au layer $(\sim 100 \mathrm{~nm})$. On the patterned glass substrates, a PEDOT:PSS dispersion (Baytron $\mathrm{P}$, Bayer AG) was spin cast in ambient air (layer thickness: $4.8 \times 10^{2} \mathrm{~nm}$ as determined by a Tencor P10 surface profiler). For the electrical measurements, the polymer layer was dried under a vacuum and placed into a sealed chamber in a nitrogen atmosphere to prevent extensive wetting of the film. In order to improve the electrical contact between the gold electrodes and the wiring, indium was applied. Optical measurements were performed in air on the complete devices.

Two types of electrode geometries were used. The first is a linear four-probe geometry, were infrared radiation is applied between the middle two probe electrodes (labeled 3 and $4)$. The active area between the probe electrodes is $2.7 \times 4$ $\mathrm{mm}$. A constant current is applied via the outer electrodes (1 and 2) and the resistance of the film can be determined by measuring the voltage over the middle two probe electrodes. The change in voltage over 3 and 4 is measured upon illumination between 3 and 4 . In the second type of device, the electrodes are positioned in such a way that one obtains a Wheatstone bridge (see next).

\section{B. Opto-electrical measurements}

The effect of infrared radiation on the conductivity of the doped $\pi$-conjugated polymer film was studied using the linear four-probe geometry just described. In the frequency domain experiments, the infrared light (from a tungsten halogen source with RG830 low-pass filter) was modulated using a mechanical chopper. The variation in the voltage over electrodes 3 and 4 induced by the illumination was measured using phase-sensitive detection (Stanford Research 830 DSP lock-in amplifier). A constant current was passed through the devices using a Keithley 2400 source meter. The small changes in resistivity of the polymer film induced by the infrared light can also lead to a small change in the current flowing through the device. As the voltage over the probe electrodes is equal to the product of current and resistance, the change in current may also give a contribution to the modulation of the voltage. The transient response time of the current source to a sudden change in the load resistance is specified at $30 \mu \mathrm{s}$. Therefore, for modulation frequencies of the infrared light $<10 \mathrm{kHz}$, effects due to a change in current can be neglected. In the time domain experiments, coherent radiation $(1047 \mathrm{~nm})$ from a miniature $Q$-switched Nd:YLF laser (Spectra Physics Model 7960L3S) was used as infrared source. Voltage transients were recorded using a Tektronix DPS 3052 oscilloscope in the ac mode.

\section{Near steady-state photoinduced absorption}

Photoinduced absorption (PIA) spectra were recorded using the Nd:YLF laser as excitation source. The change in transmission of a "white" probe light from the tungstenhalogen source through the sample $(\Delta T)$ was recorded after dispersion by a grating monochromator. For detection, either a Si or InGaAs photodiode was used. The pump power incident on the sample was typically $25 \mathrm{~mW}$ with a beam diameter of $2 \mathrm{~mm}$. The component of the signal from the photodiodes at the frequency corresponding to the modulation of the pump source, was recorded using a lock-in amplifier. The PIA $(-\Delta T / T \approx \Delta \alpha d)$ is directly calculated from the change in transmission after correction for stray light from the excitation source, which is recorded in a separate experiment. PIA spectra are recorded with the pump beam in a direction almost parallel to the direction of the probe beam.

\section{Transient sub-picosecond photoinduced absorption}

The femtosecond laser system used for pump-probe experiments consists of an amplified Ti:Sapphire laser (Hurricane, Spectra Physics). Pulses from a mode-locked Ti:Sapphire oscillator are amplified in a Nd:YLF pumped regenerative amplifier using chirped pulse amplification. The amplifier provides $150 \mathrm{fs}$ pulses at $800 \mathrm{~nm}$ with an energy of $750 \mu \mathrm{J}$ and a repetition rate of $1 \mathrm{kHz}$. For the experiment, pump pulses in the infrared region are created via optical parametric amplification (OPA) of the $800 \mathrm{~nm}$ pulse by a $\beta$-bariumborate (BBO) crystal. A phase locked mechanical chopper is placed in the pump beam to block every other pump pulse so that repetition rate of the excitation pulses is $500 \mathrm{~Hz}$. The pump beam was focused to a spot size of about $2 \mathrm{~mm}^{2}$ with a fluence of $0.1 \mathrm{~mJ} / \mathrm{cm}^{2}$ per pulse. The probe pulses are generated in a separate optical parametric amplifier also pumped by the Ti:Sapphire amplifier. The pulses have a repetition rate of $1 \mathrm{kHz}$ and were reduced in intensity compared to the pump beam using neutral density filters. The probe beam was linearly polarized at the magic angle of $54.7^{\circ}$ with respect to the pump polarization in order to cancel out orientation effects in the measured dynamics. The probe pulses could be delayed with respect to the pump using a motorized translation stage. The differential transmission was obtained by selecting the $500 \mathrm{~Hz}$ component in the output of an InGaAs photodiode monitoring the probe pulses, using a lock-in amplifier.

\section{RESULTS}

The effect of infrared radiation on the resistance of the doped $\pi$-conjugated polymer film was studied using a device with a linear four-probe electrode geometry. Typical results are shown in Fig. 1. In these measurements, the dc component of the voltage over the probe electrodes is close to $9 \mathrm{~V}$, corresponding to a dark resistance $R$ of $1 \mathrm{M} \Omega$. The modulated illumination results in a small alteration of the voltage on the order of a few hundred microvolts, with the exact magnitude depending on the frequency of the modulation. The amplitude of the alternating component of the voltage $\left(V_{\mathrm{ac}}\right)$ is shown in part a, while part b illustrates the phase angle between $V_{\text {ac }}$ and the illumination. The amplitude of the radiation- induced modulation of the voltage was found to depend linearly on the current applied through the device 


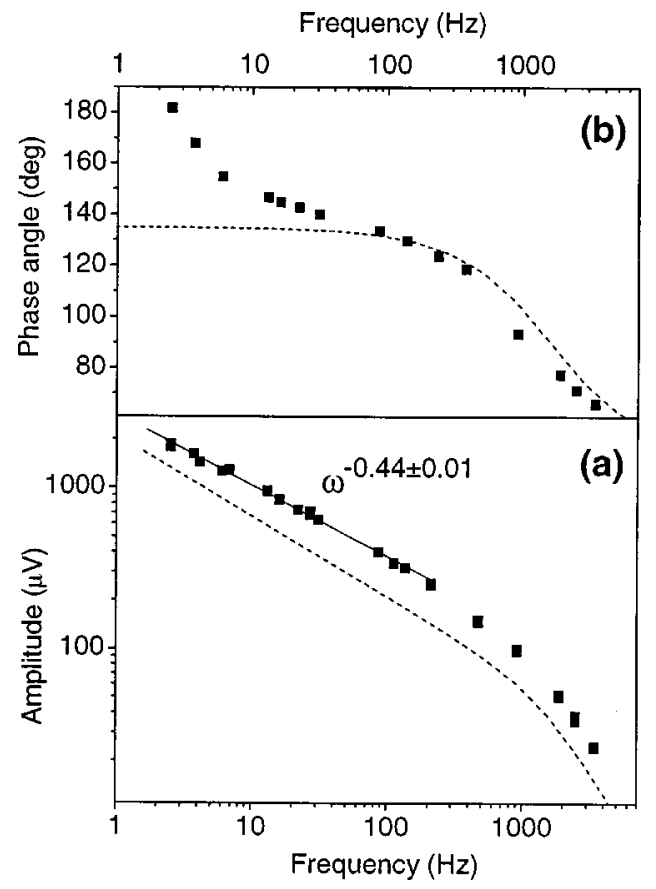

FIG. 1. (a) Amplitude of the ac component of the voltage induced by modulated illumination between the probe contacts as a function of modulation frequency. A current of $11 \mu \mathrm{A}$ through the PEDOT:PSS film is applied. Illumination comes from a tungsten-halogen source with $\lambda>830 \mathrm{~nm}$ lowpass filter, delivering $\sim 25 \mathrm{~mW}\left(0.4 \mathrm{~W} / \mathrm{cm}^{2}\right)$ at the device. The solid line indicates the results of a linear regression to the datapoints in the range of $3-200 \mathrm{~Hz}\left(\omega^{-0.44 \pm 0.01}\right)$. The dashed line represents a plot of Eq. (14) with $k=1 / \mathrm{RC}=(0.1 \mathrm{~ms})^{-1}$. (b) Phase angle of the photomodulated voltage as a function of frequency. Dashed line: Eq. (14) with $k=(0.1 \mathrm{~ms})^{-1}$ and $d R / d \mathcal{T}=3 \times 10^{2} \Omega / \mathrm{K}$.

(data not shown). The phase angle of $V_{\text {ac }}$ shows the expected change of $180^{\circ}$ when the current is reversed. At $10 \mathrm{~Hz}$, the amplitude of $V_{\text {ac }}$ amounts to $1 \mathrm{mV}$. Taking into account the square wave modulation and the root-mean-square output of the lock-in detector this implies that the change in resistance divided by the dark resistance, $\Delta R / R$, amounts to 0.3 $\times 10^{-3}$.In the frequency range of $2-300 \mathrm{~Hz}$, the amplitude is proportional to $\omega^{-\alpha}$ with $\alpha=0.44 \pm 0.01$. The value of the exponent has been determined from a linear regression.

At frequencies $>300 \mathrm{~Hz}$, the amplitude shows a steeper dependence on the modulation frequency. Also shown is the phase angle as a function of frequency. If we look at, say, $100 \mathrm{~Hz}$, we find a phase angle of $135^{\circ}$. This shows that the electrical signal lags behind in phase relative to the ir light by about $45^{\circ}$. The findings indicate that irradiation results in a change of the resistivity of the film that only relaxes quite slowly back to its dark level. In the range of 10 to $200 \mathrm{~Hz}$, the phase angle shows a weak dependence on the frequency with a value close to $135^{\circ}$ or $-45^{\circ}$, depending on the direction of the current. Above $300 \mathrm{~Hz}$, the phase lag between voltage and illumination rapidly increases. At frequencies below $10 \mathrm{~Hz}$, we also observe a strong dependence of the phase angle on frequency.

Figure 2 illustrates the results of an experiment in which the dependence of the voltage modulation on the intensity of the applied infrared radiation was studied. We find that the amplitude of the voltage is almost linear in the intensity $I$ of

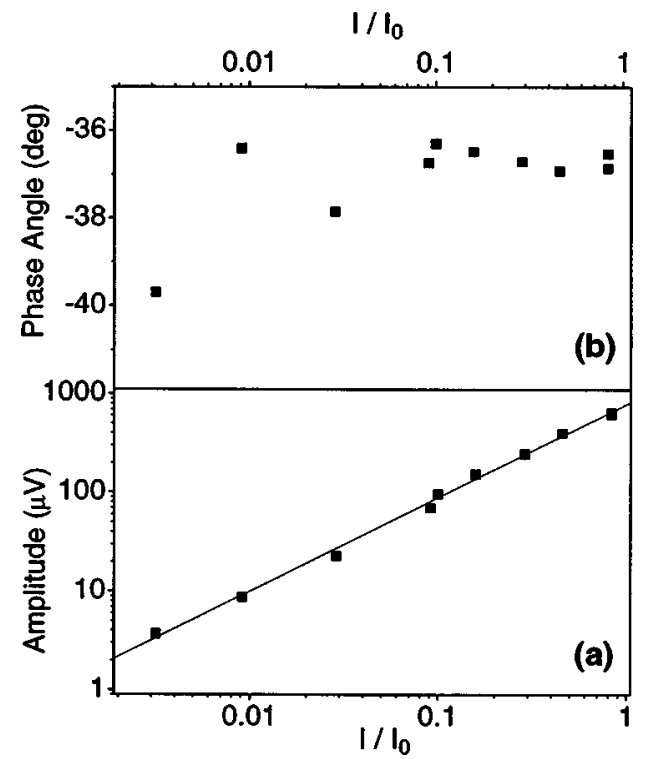

FIG. 2. (a) Intensity dependence of the amplitude of the ac component of the voltage over the probe electrodes of the device under modulated illumination. Current: $9 \mu \mathrm{A}$. Illumination: tungsten halogen with $\lambda>830 \mathrm{~nm}$ lowpass filter, delivering $I_{0}=0.4 \mathrm{~W} / \mathrm{cm}^{2}$ at the device. Mechanical modulation at $17.5 \mathrm{~Hz}$. The straight line represents a linear regression yielding $|\Delta V(I)| \propto I^{0.95 \pm 0.02}$. (b) Phase angle of the ac component of the voltage corresponding to the amplitude shown in (a).

the illumination $\left(\propto I^{\alpha}\right.$ with $\alpha=0.95 \pm 0.02$ at $17.5 \mathrm{~Hz}$ modulation). Also shown is the dependence of the phase angle on intensity. Interestingly, the phase lag is virtually independent of the intensity of illumination. This indicates that the mechanism by which resistivity relaxes back to its dark level does not change as a function of the intensity of illumination.

The measurements shown in Fig. 2 also pertain to illumination with a tungsten-halogen lamp. When light of 1.18 $\mathrm{eV}(1047 \mathrm{~nm})$ from a pulsed Nd:YLF laser was used to illuminate the device, we found that the dependence of the voltage amplitude on the intensity can be described by $\left|V_{\text {ac }}(I)\right|$ $\propto I^{\alpha}$ with $\alpha=1.0 \pm 0.03$. This holds for both $274 \mathrm{~Hz}$ and 624 $\mathrm{Hz}$ chopping rates, with the incident power varying between 4 and $400 \mathrm{~mW} / \mathrm{cm}^{2}$. For both chopping rates, no significant dependence of the phase angle on the intensity could be detected. The temporal width of the laser pulses used is about $10 \mathrm{~ns}$. When even shorter pulses from a Ti:Sapphire laser are used ( $\sim 200 \mathrm{fs}, 1450 \mathrm{~nm}$ wavelength), we do find deviations from linearity when studying the dependence of the voltage response on the intensity of the incoming light (see Fig. 3). At intensities close to $0.4 \mathrm{~W} / \mathrm{cm}^{2}(23 \mu \mathrm{J} /$ pulse, $1 \mathrm{kHz}$ rep rate) the amplitude of the voltage deviates from the linear behavior observed at lower intensities. The phase angle is still found to be independent of the light intensity. This may indicate that the deviation from the linear dependence of the voltage on intensity results mainly from nonlinearity in the absorption of radiation and not primarily from a change in relaxation mechanism.

The studies in the frequency domain, as illustrated in Fig. 1, have been complemented by studies in the time domain using pulsed irradiation from the Nd:YLF laser. Typical results are illustrated in Fig. 4. As can be seen, the infrared pulse results in a rapid decrease of the voltage over the probe 


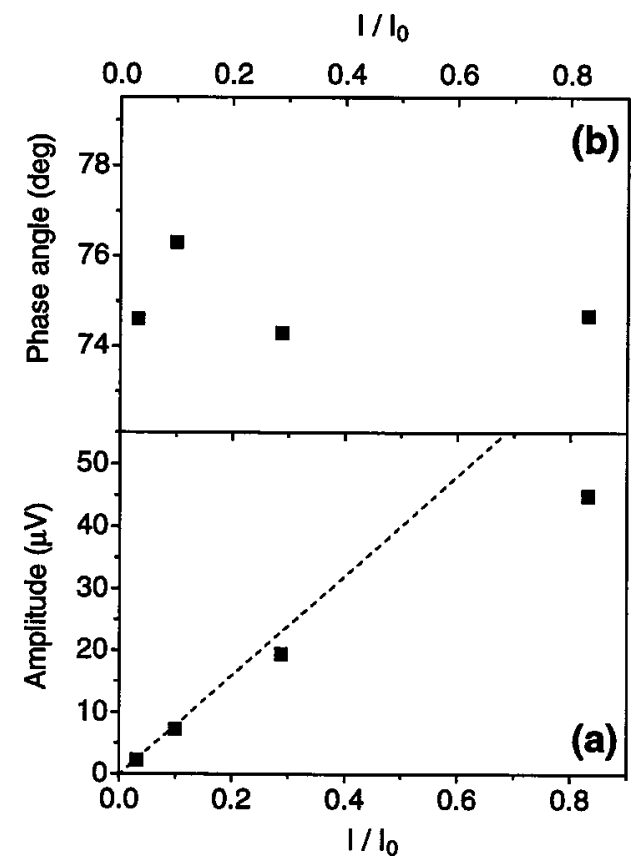

FIG. 3. Amplitude (a) and phase angle (b) of the ac component of the voltage over the probe electrodes induced by pulsed illumination at $0.86 \mathrm{eV}$ from a Ti:Sapphire pumped optical parametric oscillator, delivering $I_{0}$ $=0.4 \mathrm{~W} / \mathrm{cm}^{2}$ with a fluence $23 \mu \mathrm{J} /$ pulse.

electrodes, indicating a rapid drop in the resistance of the film. Shown in the inset B of Fig. 4 is the early time response of the device and, as can be seen, the lowering of the voltage occurs on the microsecond time scale. A fit of an exponential function to the drop of the voltage yields a time constant of $8 \mu$ s. As will be discussed next, we attribute this rise to a finite RC time of the combination of device and oscilloscope. After the rapid initial drop, part of the voltage is quickly recovered but also a particularly slow decay component can be discerned in the time trace. From a log-log plot of the

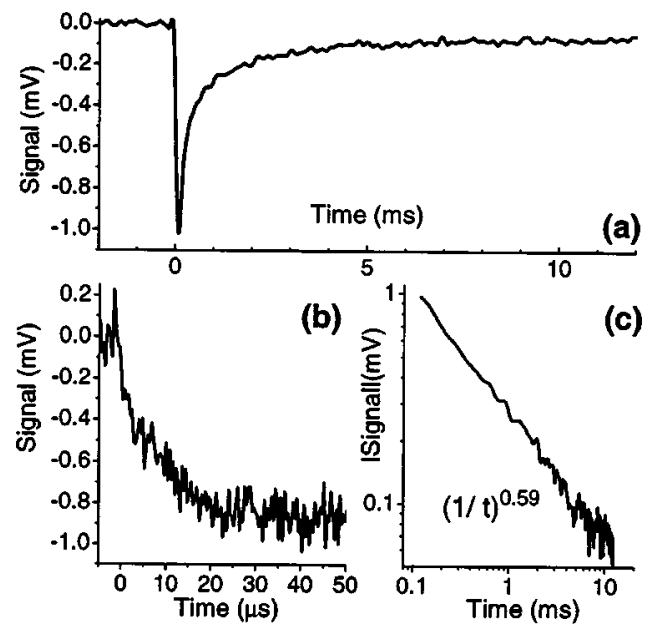

FIG. 4. (a) Change in voltage over the probe electrodes upon pulsed illumination with $1.18 \mathrm{eV}$ from a Nd:YLF laser. Pulse length $10 \mathrm{~ns}$, pulse repetition rate $274 \mathrm{~Hz}$, and fluence $30 \mu \mathrm{J} /$ pulse. Current through polymer layer: $5 \mu \mathrm{A}$. (c) Expanded view covering the rapid drop in voltage. (c) Double logarithmic plot of the absolute value of the change in voltage vs time.

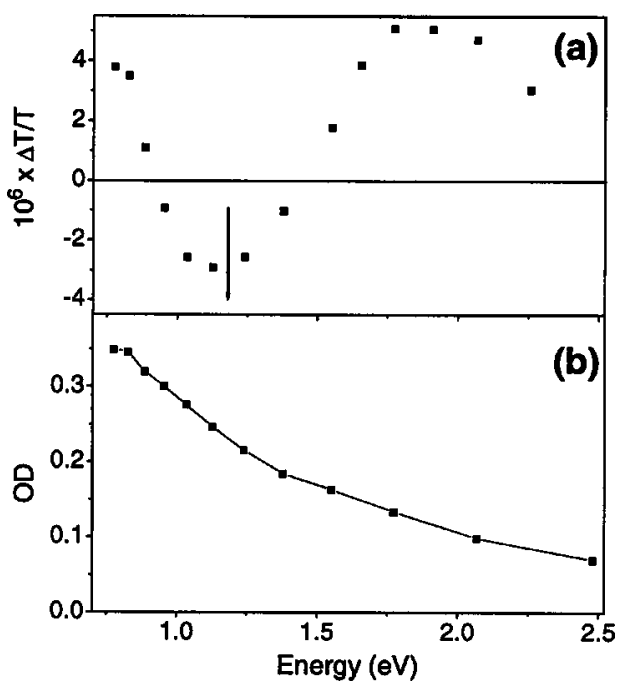

FIG. 5. (a) Photoinduced absorption of the PEDOT:PSS polymer layer, induced by $1.18 \mathrm{eV}$ excitation. Relative change in transmission $(\Delta T / T)$ is given for various probe wavelengths. Excitation source pulsed Nd:YLF laser delivering $0.4 \mathrm{~W} / \mathrm{cm}^{2}$ with $517 \mathrm{~Hz}$ repetition rate. (b) Absorbance of the same film.

absolute value of the signal (see inset $\mathrm{C}$ ), a power-law decay of the change in voltage with $|\Delta V(t)| \propto(1 / t)^{\alpha}$ with $\alpha=0.59$ \pm 0.01 becomes evident.

In summary, irradiation of the polymer film through which an electric current is flowing results in a drop (in the absolute sense) of the voltage between two probe electrodes positioned on the sides of the illuminated area. The drop in voltage can be understood in terms of a lowering of the resistance. To characterize the infrared induced changes in the polymer film further, we have also performed some optical studies. In particular, we have looked for changes in the optical absorption spectrum of the polymer film upon irradiation with infrared light. In these optical experiments, no electrical current was flowing through the device.

In Fig. 5, some results of the PIA measurements are shown. In these experiments, the device is irradiated with pulses of $1.18 \mathrm{eV}$ photons coming from the Nd:YLF laser and the change in the transmittance $(T)$ of the device for light from a continuous tungsten-halogen source is measured. The changes in the transmittance $(\Delta T)$ induced by the infrared excitation, divided by the transmittance $T$ are plotted, (see part A). For small values of $\Delta T / T$, the relationship $\Delta T / T=\Delta A$ holds, with $\Delta A$ as the change in absorbance due to excitation. For comparison, the absorbance $A$ of the polymer film is also shown (part B). The measurements reveal a bleaching of the absorption band of the doped polymer in the infrared region $(\Delta T>0)$, accompanied by an increased absorption of light in the near-infrared and visible parts of the spectrum $(\Delta T<0)$. In Fig. 6 , the frequency dependence of the PIA signals is illustrated for detection with photon energies of 0.83 and $1.77 \mathrm{eV}$. The dependence has been analyzed in terms of a power law $|\Delta T(\omega)| \propto(1 / \omega)^{\alpha}$. This yields exponents $\alpha$ of 0.4 and 0.6 for probe photon energies of 0.83 and 1.77 , respectively.

Using the pump-probe technique, we have also studied the optical transients in the sub-ps time domain. In Fig. 7, 


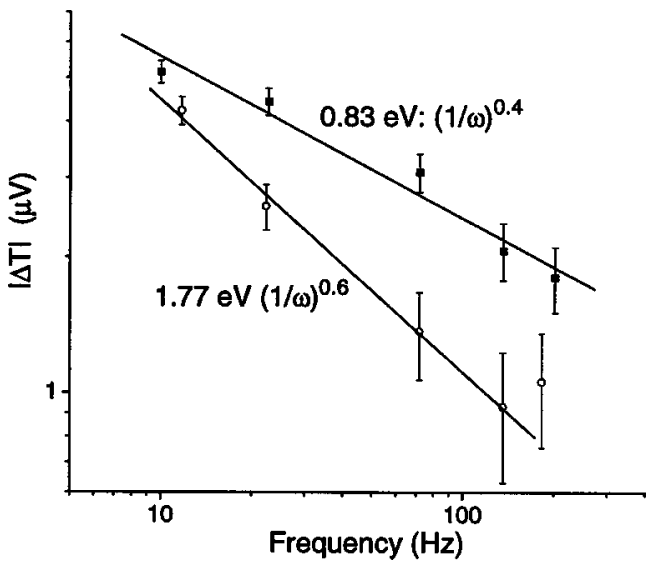

FIG. 6. Frequency dependence of the photoinduced absorption signal of the PEDOT:PSS film probed at $0.83 \mathrm{eV}$ and $1.77 \mathrm{eV}$. Excitation source: pulsed $\mathrm{Nd}$ :YLF laser with $1.18 \mathrm{eV}$ photon energy and $4087 \mathrm{~Hz}$ repetition rate followed by a mechanical chopper.

measurements of the relative differential transmission $\Delta T(t) / T$ using pump and probe photon energies of 0.86 and $0.94 \mathrm{eV}$, respectively, are shown. As can be seen, there is a very rapid response (full width at half maximum $\sim 0.6 \mathrm{ps}$ ), which is close in temporal width to the instrument response function. The positive $\Delta T / T$ values indicate a bleaching of the absorption at the probe wavelength. A major part of the induced bleaching decays very rapidly. However, there is a smaller component that decays more slowly. The inset shows the $\Delta T / T$ signal in a double logarithmic plot. For times $>1$ $\mathrm{ps}$, the decay of the $\Delta T / T$ signal can be described by a power law with $|\Delta T(t) / T| \propto(1 / t)^{\alpha}$ and an exponent $\alpha$ of 0.65 \pm 0.01. In Fig. 8, we show optical transients obtained for various probe photon energies. The pump photon energy was kept constant at $0.86 \mathrm{eV}$. For all probe energies used $(0.86$, $0.88,0.90$, and $1.00 \mathrm{eV}$ ), positive $\Delta T / T$ values are observed upon excitation, signifying a bleaching of the absorption upon excitation. For $1.0 \mathrm{eV}$, the magnitude of the rapid component to $\Delta T / T$ seems reduced. These results may be com-

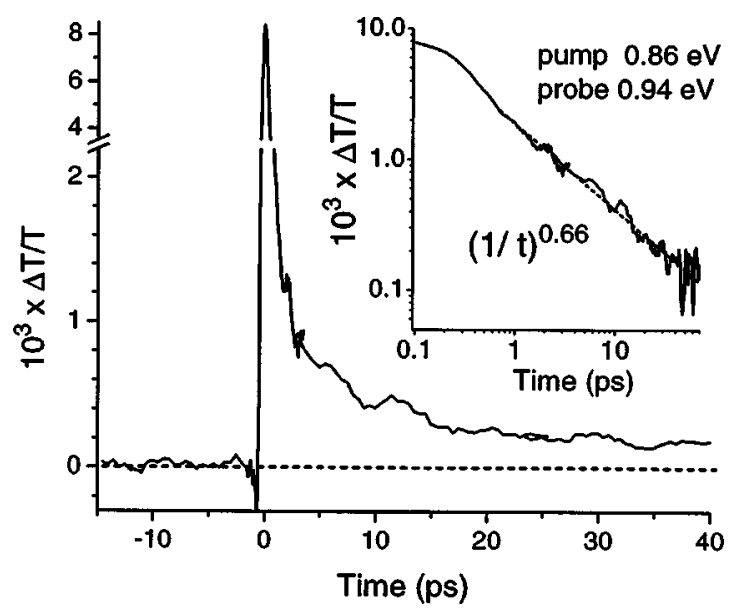

FIG. 7. Differential transmission $\Delta T(t)$ of the PeDOT:PSS film induced by a pump beam with photon energy $0.86 \mathrm{eV}$, repetition rate $500 \mathrm{~Hz}$, fluence 3 $\mu \mathrm{J} /$ pulse, and intensity $33 \mathrm{~mW} / \mathrm{cm}^{2}$. Probe photon energy $0.94 \mathrm{eV}$. The insert shows the same signal in a log-log plot and the dashed line represents a linear regression, yielding $|\Delta T(t)| \propto(1 / t)^{0.65 \pm 0.01}$.

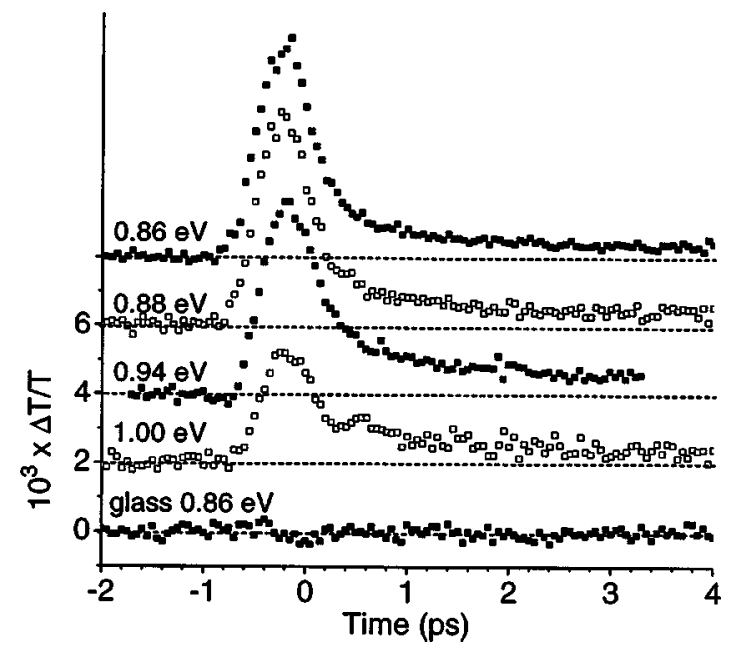

FIG. 8. Photoinduced absorption transients for the PEDOT:PSS film induced by a pump beam with photon energy $0.86 \mathrm{eV}$, and various probe energies. Pump repetition rate $500 \mathrm{~Hz}$ and fluence $3 \mu \mathrm{J} /$ pulse. For comparison, the response of just the glass substrate is also shown.

pared to those in Fig. 5. From Fig. 5, it can be observed that $\Delta T / T$ changes sign when going from 0.86 to $1.0 \mathrm{eV}$.

To evaluate the feasibility of a detector for infrared radiation using films of doped $\pi$-conjugated polymer, we have constructed devices following the classical bolometer design. This is illustrated in Fig. 9. Electrodes 1 and 2 are used to apply a current and two separate lanes for the current are created by scratching away the polymer film in the middle of the device (hatched area). Electrodes 3 and 4, which divide both lanes into two equal parts, are used as probes. Illumination between, say, electrodes 1 and 3 will cause an unbalance in the resistance bridge and will result in a voltage over 3 and 4. In order to adjust $V_{34}$ to zero in the dark, a variable resistance between 1 and 4 (or 2 and 4 ) is applied. For illumination at $1.18 \mathrm{eV}$ photon energy with power $\geqslant 1.6 \mathrm{~mW}$,

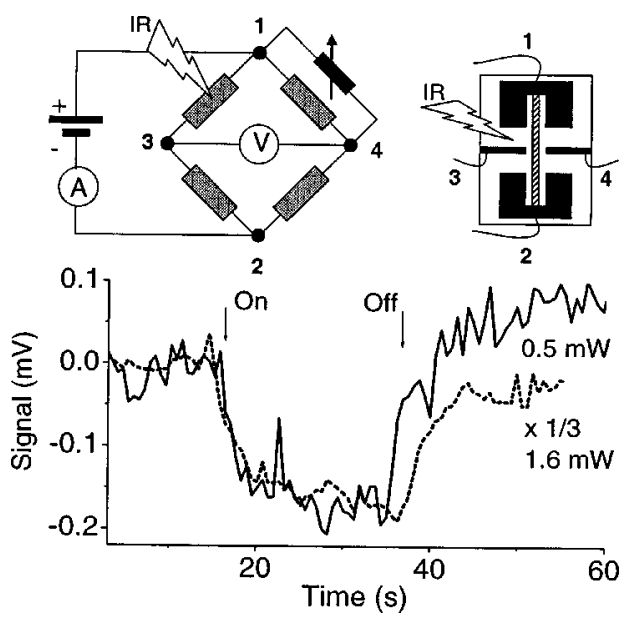

FIG. 9. Voltage output of a PEDOT:PSS film with electrodes in bolometer configuration. Excitation by pulsed Nd:YLF laser at $5000 \mathrm{~Hz}$ repetition and $1.18 \mathrm{eV}$ photon energy. Solid line: irradiation with $0.5 \mathrm{~mW}\left(8 \mathrm{~mW} / \mathrm{cm}^{2}\right)$. Dashed line: $1.6 \mathrm{~mW}\left(26 \mathrm{~mW} / \mathrm{cm}^{2}\right)$ with output scaled by a factor $1 / 3$. Current applied via electrodes 1 and 2: $0.5 \mu \mathrm{A}(3.5 \mathrm{~V})$. The drawing on the right-hand side shows a schematic layout of the electrode geometry. The vertical hatched strip indicates where the polymer has been removed by scratching. The left-hand side drawing illustrates the electrical setup. 
$V_{34}$ is clearly above the noise level when measured with a dc voltmeter (integration time for one measurement $20 \mathrm{~ms}$ ). For $0.5 \mathrm{~mW}$, the voltage output $(0.2 \mathrm{mV})$ approaches the noise level. For these intensities, also the stability of the baseline starts to become an important factor. The baseline fluctuations most likely have their origin in dynamical processes in the polymer film.

\section{DISCUSSION}

The experimental data presented herein show that illumination of the PEDOT:PSS film with infrared light changes its resistance. This phenomenon could be described as photoconduction and can be due to several causes. First, it could result from photogeneration of charge carriers and, second, it may result from a light-induced change in mobility of carriers already present in the material. In the case of the oxidatively doped PEDOT polymer with a high density of charge carriers $\left(10^{19} \mathrm{~cm}^{-3}\right)$, a contribution of photogenerated charge carriers to the transient conductivity is unlikely for the following reasons. Recent investigations of PEDOT show that the band gap of the fully undoped material is $>1.5$ eV. ${ }^{15,17,22}$ In our case, the photon energy would not be enough to generate carriers via one photon processes. Charge generation involving more than one photon can be excluded on the grounds that the photoconduction is linear in the intensity of the light applied. Even if an oppositely charged pair of carriers was generated from an absorbed photon, it is to be expected that the negatively charged carrier would recombine very quickly with one of the holes that are present in the material with a high density. Such a recombination event would reduce the yield of photogenerated carriers to zero. Therefore, we conclude that a contribution from photogenerated charges to the photoinduced conduction is unlikely and that the effect results from a modification of the mobility of carriers present in the material induced by light.

Irradiating the polymer film with infrared light at a wavelength where the film absorbs light will eventually result in a heating of the absorbing layer. One of the typical features of disordered polymeric conductors near room temperature is that the electrical (sheet) resistance of films of these materials shows a decrease with increasing temperature. This has also been observed for PEDOT. ${ }^{12}$ It is therefore very likely that the heating of the polymer film due to the infrared radiation makes a contribution to the optically induced lowering of the resistance of the film. When this mechanism is operative, the relaxation of the resistance back to its normal "dark" level may be controlled by the heat transport from the polymer film to its surroundings.

Following Smith et al., ${ }^{23}$ we analyze the heat transport from the polymer film to its substrate. We assume that the cooling of the polymer film is solely due to the conduction of heat to the supporting glass substrate. Furthermore, it is assumed that the polymer layer is infinitely thin with a uniform temperature. When the illuminated area is large compared to the thickness of the device, one may reduce the heat transport problem to one dimension. If the power of the incoming radiation is sinusoidally modulated with angular frequency $\omega$ and amplitude $W_{0}$ (in $\mathrm{J} / \mathrm{s}$ ), we may write for the change in temperature $(\Delta \mathcal{T})$ of the polymer film under influence of the radiation:

$$
C \frac{d \Delta \mathcal{T}}{d t}+W_{\text {out }}=W_{0} e^{i \omega t}
$$

where $C$ is the thermal capacity and $W_{\text {out }}$ is the rate of flow out of the polymer film due to heat conduction. When the polymer film is very thin, its heat capacity may be neglected. If we neglect heat losses due to radiation and through contact with air and take into account only the heat transport to the supporting substrate then Fick's first law of diffusion can be applied:

$$
W_{\text {out }}=-S K\left(\frac{\partial(\Delta \mathcal{T})}{\partial y}\right)_{y=0}=W_{0} e^{i \omega t},
$$

where $S$ is the surface area and $K$ is the thermal conductivity. It is assumed that the illuminated area is large compared to the thickness of the layer so that the heat diffusion can be regarded as a one-dimensional problem. The direction of the $y$ axis is normal to the film. The surface $y=0$ is taken to coincide with the interface between the polymer film and the glass substrate. The diffusion of heat within the glass support is governed by the equation for the heat diffusion in one dimension:

$$
\frac{\partial}{\partial t}=a \frac{\partial^{2}(\Delta \mathcal{T})}{\partial y^{2}},
$$

where $a=\frac{K}{s \rho}$ with $s$ denoting the specific heat and $\rho$ as density of the glass. We look for solutions of the form:

$$
\Delta \mathcal{T}(\omega, y, t)=\phi(y) \Delta \mathcal{T}(\omega, y=0) .
$$

We assume that all the radiation is absorbed in the infinitely thin layer of polymer on the $y=0$ surface of the glass substrate. The thickness $l$ of the supporting glass plate is assumed to be so large that at the back surface of the plate at $y=l$, no appreciable heating of the glass occurs. We can summarize these statements by writing the following boundary conditions for the heat diffusion equation.

$$
\begin{cases}\phi(y)=1 & \text { for } y=0 \\ \phi(y)=0 & \text { for } y=l .\end{cases}
$$

Equation (4) can now be rewritten as

$$
\frac{\partial^{2} \phi}{\partial y^{2}}=m^{2} \phi \quad \text { with } \quad m=\sqrt{\frac{i \omega}{a}}
$$

general solutions are

$$
\phi(y)=B \sinh (m y+b)+C \cosh (m y+c),
$$

and a particular solution obeying the boundary conditions is

$$
\Delta \mathcal{T}(\omega, y)=\Delta \mathcal{T}(\omega, y=0) \frac{\sinh \left(\sqrt{2 i}(l-y) / l_{0}\right)}{\sinh \left(\sqrt{2 i}\left(l / l_{0}\right)\right)},
$$

where

$$
l_{0}=\sqrt{\frac{2 a}{\omega}} .
$$


We now look for an expression of $\Delta \mathcal{T}(\omega, y=0)$. From Eqs. (2) and (8), it follows that

$$
\Delta \mathcal{T}(\omega, y=0)=\frac{l_{0} W_{0}}{S K \sqrt{2 i}} e^{i \omega t} \tanh \left(\sqrt{2 i}\left(l / l_{0}\right) .\right.
$$

For small changes in temperature $(\Delta \mathcal{T})$, the change in electrical resistance $(\Delta R)$ may be assumed to be linear in $\Delta \mathcal{T}$. One can then write

$$
\Delta R(\omega)=\left(\frac{d R}{d}\right)_{\mathcal{T}=\mathcal{T}_{0}} \frac{l_{0} W_{0}}{S K \sqrt{2 i}} e^{i \omega t} \tanh \left(\sqrt{2 i}\left(\ell / l_{0}\right)\right) .
$$

For ordinary glass, we have density $\rho=2.5 \times 10^{3} \mathrm{~kg} \mathrm{~m}^{-3}$, specific heat $s=0.8 \times 10^{3} \mathrm{~kg}^{-1} \mathrm{~K}^{-1} \mathrm{~J}$, thermal conductivity $K=0.93 \mathrm{~J} \mathrm{~s}^{-1} \mathrm{~m}^{-1} \mathrm{~K}^{-1}$. From these numbers, we calculate a value for $l_{0}$ of $0.1 \mathrm{~mm}$ at $100 \mathrm{~Hz}$ modulation frequency. The glass substrates used are approximately $1 \mathrm{~mm}$ thick. For sufficiently thick substrates $\left(l \gg l_{0}\right)$, Eq. (11) reduces to

$$
\begin{aligned}
\Delta R(\omega) & =\left(\frac{d R}{d \mathcal{T}}\right)_{\mathcal{T}=\mathcal{T}_{0}} \frac{l_{0} W_{0}}{S K \sqrt{2 i}} e^{i \omega t} \\
& =\frac{W_{0}}{S}\left(\frac{d R}{d \mathcal{T}}\right)_{\mathcal{T}=\mathcal{T}_{0}} \sqrt{\frac{1}{2 s \rho K}} \frac{e^{i \omega t}}{\sqrt{\omega}}(1-i) \quad l \gg l_{0} .
\end{aligned}
$$

Alternatively, one may also define a critical frequency $\omega_{0}$ :

$$
\omega_{0}=\frac{2 K}{l^{2} s \rho} .
$$

For $\omega \gg \omega_{0}$, the temperature at the back surface of the glass substrate at $y=l$ will show a negligible variation with the irradiation, so that Eq. (12) applies. For a $1 \mathrm{~mm}$ glass plate, we calculate $\omega_{0}=0.9 \mathrm{rad} / \mathrm{s}=0.14 \mathrm{~Hz}$.

From Eq. (12), we can derive the following three predictions: (1) The change in resistance will be linear in the applied optical power; (2) The electrical signal will lag behind the optical input with a phase angle difference of $45^{\circ}$. This phase angle is independent of the power applied; and (3) The amplitude of the change in resistance $|\Delta R(\omega)|$ follows a $1 / \sqrt{ } \omega$ dependence on the modulation frequency. Fourier transformation of this frequency dependence leads to a $1 / \sqrt{ } t$ dependence for the change in resistance on time $t$. What is interesting about this time dependence is that it does not have a characteristic time; the plot of $1 / \sqrt{ } t$ versus $t$ looks the same when normalized and plotted on various time scales. The other interesting feature is the singularity at $t=0$ in the time domain. In an experimental setup, however, the combination of device and detection apparatus will always show a finite $\mathrm{RC}$ time. Therefore, in the time domain, the response of the polymer film has to be convoluted with the exponential response curve of the detection with time constant $k$ $=1 / \mathrm{RC}$ and this will remove the singularity at $t=0$. In the frequency domain, the response of the film has to be multiplied by the Lorentzian describing the $\mathrm{RC}$ response of device and detection. This gives

$$
\begin{aligned}
\Delta R(\omega)= & \frac{W_{0}}{S} \sqrt{\frac{1}{2 s \rho K}}\left(\frac{d R}{d \mathcal{T}}\right)_{\mathcal{T}=\mathcal{T}_{0}} \frac{e^{i \omega t}}{\sqrt{\omega}}\left[\left(\frac{k}{\omega^{2}+k^{2}}\right.\right. \\
& \left.\left.-\frac{\omega}{\omega^{2}+k^{2}}\right)-i\left(\frac{\omega}{\omega^{2}+k^{2}}+\frac{k}{\omega^{2}+k^{2}}\right)\right] .
\end{aligned}
$$

These predictions can now be compared with the experimental data. Taking the materials parameters for glass, one can calculate the amplitude of the temperature variations for illumination with $0.4 \mathrm{~W} / \mathrm{cm}^{2}$ irradiation. This amounts to 0.1 $\mathrm{K}$ for modulation at $10 \mathrm{~Hz}$. In order to account for the observed voltage modulation, one needs to assign a value to $(d R / d \mathcal{T})$ of $7 \times 10^{2} \mathrm{ohm} / \mathrm{K}$. Thus, $(d R / d \mathcal{T})(1 / R)$ should be on the order of $10^{-3}$. Experimentally, we find that the normal dark resistance of the PEDOT:PSS is reduced when the temperature is raised. For the temperature range of 273 to $303 \mathrm{~K}$, we find that the relative change in resistance, $\Delta R / R$, is indeed on the order of $10^{-3}$ per $\mathrm{K}$.

As shown in Fig. 1, the dependence for the amplitude of the voltage modulation is $(1 / \omega)^{0.44}$ in the frequency range of 3 to $300 \mathrm{~Hz}$. At higher frequencies, a deviation occurs. The dashed line in Fig. 1 represent a plot of the amplitude $|\Delta R(\omega)|$ from Eq. (14). In this plot, a constant $k=1 / \mathrm{RC}=1 /$ $(0.1 \mathrm{~ms})$ was used and $(d R / d \mathcal{T})$ was taken to be $3 \times 10^{2}$ $\mathrm{ohm} / \mathrm{K}$. It thus seems that the deviation of the signal at higher frequencies can be understood in terms of a finite RC time of the combination of device and detection system. About the origin of this RC time, we can say the following. The lock-in amplifier has a capacitance of $25 \mathrm{pF}$ and an impedance of $10 \mathrm{M} \Omega$. The resistance over the probe electrodes is $2 \mathrm{M} \Omega$. In combination with the $25 \mathrm{pF}$ capacitance, this gives an RC time of $0.05 \mathrm{~ms}$, which is only a factor of 2 short of the experimental observation.

In the range $10-100 \mathrm{~Hz}$, the phase angle of the signal is close to $-45^{\circ}$ or $135^{\circ}$, in good agreement with Eq. (12) [see Fig. 1(B)]. At higher frequencies, the phase angle shows a dispersion. The dashed line in the plot shows the prediction based on Eq. (14) and it seems that the deviation at high frequency can be understood in terms of a finite RC time of the combination of device and detection. At low frequency, a change in phase angle occurs also. At this moment, we do not have an explanation for this. It may be that the assumption $\left(\ell \gg l_{0}\right)$ used to derive Eq. (12) breaks down in this region. Thus, we conclude that the dependence of the amplitude of the voltage modulation is given by a power law $(1 / \omega)^{0.44}$. After Fourier transformation, one obtains a $(1 / t)^{\alpha}$ dependence with $\alpha=1-0.44=0.56$.

From the studies in the time domain (Fig. 4), we find that the time dependence of the amplitude as observed for $t$ $>10 \mu \mathrm{s}$ is also given by a power law $(1 / t)^{0.59}$. This is in good agreement with the frequency dependent measurements. As shown in part B of Fig. 4, we find a $8 \mu$ s rise time of the signal. This rise may be interpreted in terms of an RC time for the device+detection. The oscilloscope used has a $13 \mathrm{pF}$ capacitance and $1 \mathrm{M} \Omega$ impedance. Assuming that the impedance of the oscilloscope may be taken to be coupled in parallel to the resistance over the probe electrodes, this yield a combined resistance of $\sim 0.7 \mathrm{M} \Omega$. In combination with the 
$13 \mathrm{pF}$ capacitance, this yields an RC time of $9 \mu \mathrm{s}$. It, therefore, seems that the finite RC time observed, results mainly from the combination of capacitance in the detection and high resistance of the device.

As discussed herein, the analysis of the heating and subsequent thermal diffusion predicts a $1 / \sqrt{ } t$ time dependence whereas the experimental data show a $(1 / t)^{0.6}$ dependence and this suggests that a thermal effect is most likely the correct explanation for the changes in resistivity induced by the irradiation in the $\mu \mathrm{s}-\mathrm{ms}$ time domain.

We can make a crude estimate of the change in temperature upon irradiation with a $20 \mu \mathrm{J}$ pulse incident on a 0.1 $\mathrm{cm}^{2}$ area, similar to that used to obtain the data in Fig. 4. We assume that the pulse is completely absorbed by the $400 \mathrm{~nm}$ thick film and we take the heat capacity of the active layer to the same as for polystyrene. This yields an elevation of temperature of approximately $4^{\circ}$ right after the arrival of the pulse.

Besides this time or frequency dependence of the amplitude of the induced changes in voltage, also the predictions for the phase angle between the oscillations in the voltage and the excitation light can be compared with the experiment. Apart from the very low-frequency region where the behavior of the phase angle on frequency could not be explained (see Fig. 1), the magnitude of the phase angle observed is consistent with the interpretation. The observed independence of phase angle on the intensity of illumination is in accordance with the prediction. The proposed interpretation of the photoinduced change in resistivity in terms of a heating effect implies, however, that the optical excitations have completely thermalized within $0.1 \mathrm{~ms}$.

The measurements of the PIA of the PEDOT:PSS films give, in our opinion, additional information on the time scale at which thermalization of the excitations occurs. Let us first look at the quasisteady-state measurements as shown in Fig. 6. The frequency dependence of the infrared induced changes also indicate a power-law decay of the signals in time. At $0.83 \mathrm{eV}$ detection energy, the dependence of the PIA on frequency $\left(|\Delta T(\omega)| \propto(1 / \omega)^{0.4}\right)$ is almost similar to that observed for the change in resistance. To obtain the time dependence, one can apply a Fourier transformation yielding $|\Delta T(t)| \propto(1 / t)^{0.6}$. For detection at $1.77 \mathrm{eV}(700 \mathrm{~nm})$, the dependence is slightly different. The similarity in frequency dependence of the change in resistance and the PIA signal at infrared probe energies suggest that the optical and electrical signal have a common origin, most likely the heating of the polymer film. Due to the temperature increase, the population of the states available to the carriers will be modified and this will result in a change of the absorption spectrum.

On the sub-ns time scale, the photoinduced changes in optical transmission of the film, as detected in the infrared region, also follow an algebraic decay law $(|\Delta T(t)|$ $\left.\propto(1 / t)^{0.65}\right)$, see Fig. 7. This time dependence is similar to the one observed in quasisteady state PIA at $0.83 \mathrm{eV}$. A possible, and in our view, a likely, explanation is that also in this time domain, the photoinduced changes are due to heating and the relaxation limited by the heat transport from the film to the substrate.
On the sub-ps time scale, we observed a very rapid decay component with a decay time close to the instrument response time (see Fig. 8). This indicates the existence of relaxation processes with rate constants $<500$ fs. In our view, these processes are related to the decay of the primary photoexcitation and the dissipation of the excitation energy. Chang et al. ${ }^{14}$ have interpreted the infrared absorption band of the PEDOT in terms of the localization modified Drude model, implying that the absorption is due to motion of charge carriers in the electric field of the light. The relaxation of the associated photoexcitation can then be related to the thermalization of the charge carriers. From the modified Drude model, a relaxation time can be extracted and for PEDOT:PSS it is estimated at 1-10 fs. Such short relaxation times are compatible with the very rapid decay component which we have observed in the pump-probe measurements. The short lifetime implies a considerable homogeneous broadening of the associated optical transition of the material. For a lifetime of $1 \mathrm{fs}$, the expected homogeneous width is close to a few tenths of an $\mathrm{eV}$. The pump-probe traces for different detection wavelengths as shown in Fig. 8 give some indication of the homogenous linewidth of the electronic transition in the PEDOT:PSS. What we observe is that the bleaching band at $t=0$ is at least $0.1 \mathrm{eV}$ wide, which is much wider than the spectral width of the pump and probe pulses used. This is consistent with a homogeneous linewidth corresponding to a 1-10 fs lifetime. In analyzing the pumpprobe spectra, one has to be careful: When the pump and probe pulses overlap both in time and in space the so-called "coherent artifact" can occur. This artifact can be interpreted in terms of the exchange of energy between pump and probe beam induced by the nonlinear optical response of the medium. This implies that the positive differential transmission signals which we observe at $t=0$ can also have an origin other than a simple bleaching of the absorbance. However when the $T_{1}$ and $T_{2}$ relaxation times are very short, the contribution from such a coherent artifact is very small.

\section{CONCLUSION}

The resistance of a film of PEDOT:PSS can be modified by irradiation with infrared light. The change in resistance is attributed to a light-induced change in the mobility of the carriers associated with the (slight) heating of the polymer film due to photon absorption. The decay of the photoinduced changes in time can be understood in terms of the relaxation being limited by transport of heat from the polymer film into the supporting glass substrate. Apart from the change in resistance, an optical transient associated with the heating is also observed. Ultrafast pump-probe experiments indicate that the thermalization of the charge carriers after excitation occurs on a sub-ps time scale.

\section{ACKNOWLEDGMENTS}

The research of one of the authors (S.C.J.M.) has also been made possible by a fellowship of the Royal Dutch Academy of Arts and Sciences. The authors thank the coun- 
cil for Chemical Sciences of the Netherlands Organization for Scientific Research (CW-NWO) and the Eindhoven University of Technology for financial support through the PIONIER program.

${ }^{1}$ See, e.g., A. J. Heeger, Angew. Chem. Int. Ed. Engl. 40, 2491 (2001).

${ }^{2}$ Y. Cao, P. Smith, and A. J. Heeger, Synth. Met. 48, 91 (1992).

${ }^{3}$ F. Jonas and L. Schrader, Synth. Met. 41, 831 (1991); G. Heywang and F. Jonas, Adv. Mater. 4, 116 (1992); I. winter, C. Reece, J. Hormes, G. Heywang, and F. Jonas, Chem. Phys. 194, 207 (1995); M. Dietrich, J. Heinze, G. Heywang, and F. Jonas, J. Electroanal. Chem. 369, 87 (1994).

${ }^{4}$ L. Groenendaal, F. Jonas, D. Treitag, H. Pielarzik, and J. R. Reynolds, Adv. Mater. 12, 481 (2000).

${ }^{5}$ L. Groenendaal, G. Zotti, and F. Jonas, Synth. Met. 118, 105 (2001).

${ }^{6}$ S. M. Kogan, Sov. Phys. Semicond. 4, 1386 (1963).

${ }^{7}$ M. A. Kinch and B. V. Rollins, Br. J. Appl. Phys. 14, 672 (1963).

${ }^{8}$ H. Rohrbacher and N. Karl, Phys. Status Solidi A 29, 517 (1975); See also M. Pope and C. Swenberg, Electronic Processes in Organic Crystals (Clarendon, Oxford, 1982).

${ }^{9}$ N. Karl, in Defect Control in Semiconductors, edited by K. Sumino (Elsevier, North-Holland, Amsterdam, 1990)

${ }^{10}$ M. Iijima, G. H. Shen, Y. Takahashi, E. Fukada, A. Tanaka, and S. Sakata, Thin Solid Films 272, 157 (1996); J. C. Dubois and P. Robin, Ferroelectrics 171, 253 (1995); J. Ruth, B. R. Ratna, J. Naciri, R. Shashidhar, S. K. Prasad, D. S. Rao, and S. Chandrasekhar, Proc. SPIE 1911, 104 (1993); P. C. A. Hammes and P. P. L. Regtien, Sens. Actuators A 32, 396 (1992); H. Meixner and G. Mader, Phys. Unserer Zeit 21, 210 (1990).

${ }^{11}$ J. Kaufmann, M. G. Moss, R. E. Giedd, and T. L. Brewer, US Patent No. 5,629,665 (30 March 1997).
${ }^{12}$ A. Aleshin, R. Kiebooms, R. Menon, F. Wudl, and A. J. Heeger, Phys. Rev. B 56, 3659 (1997)

${ }^{13}$ N. F. Mott, Metal Insulator Transitions (Taylor \& Francis, London, 1990); N. F. Mott, Conduction in Non-crystalline Materials (Clarendon, Oxford, 1993).

${ }^{14}$ Y. Chang, K. Lee, R. Kiebooms, A. Aleshin, and A. J. Heeger, Synth. Met. 105, 203 (1999)

${ }^{15}$ E. E. Havinga, C. J. M. Mutsaers, and L. W. Jenneskens, Chem. Mater. 8, 769 (1996); A. Kumar, D. M. Welsh, M. C. Morvant, F. Piroux, K. A. Abboud, and J. R. Reynolds, ibid. 10, 896 (1998).

${ }^{16}$ N. F. Mott and H. Jones, The Theory of the Properties of Metals and Alloys (Dover, New York, 1958).

${ }^{17}$ J. L. Apperloo, L. Groenendaal, H. Verheyen, M. Jayakannan, R. A. J. Janssen, A. Dikhissi, D. Beljonne, R. Lazzaroni, and J.-L. Brédas, Chem.Eur. J. 8, 2384 (2002).

${ }^{18}$ R. Kiebooms, A. Aleshin, H. Hutchison, and F. Wudl, J. Phys. Chem. B 101, 11037 (1997).

${ }^{19}$ H. Bässler, Phys. Status Solidi B 175, 15 (1993)

${ }^{20}$ M. Pollak, Discuss. Faraday Soc. 59, 13 (1970).

${ }^{21}$ D. Monroe, A. C. Gossard, J. H. English, B. Golding, W. H. Haemmerle, and M. A. Kastner, Phys. Rev. Lett. 59, 1148 (1987); M. Schreiber, K. Tenelsen, and T. Vojta, J. Lumin. 66, 521 (1995); T. Wappler, T. Vojta, and M. Schreiber, Phys. Rev. B Condens. Mater 55, 6272 (1997); M. Schreiber and K. Tenelsen, J. Lumin. 58, 130 (1994).

${ }^{22}$ L. Groenendaal, G. Zotti, and F. Jonas, Synth. Met. 118, 105 (2001); F. Tran-Van, S. Garreau, G. Louarn, G. Froyer, and C. Chevrot, Synth. Met. 119, 381 (2001)

${ }^{23}$ R. A. Smith, F. E. Jones, and R. P. Chasmar, The Detection and Measurement of Infrared Radiation (Clarendon, Oxford, 1968). 